\title{
SOME COUNTEREXAMPLES RELATED TO INTEGRAL CLOSURE IN $D[[x]]$
}

\author{
BY \\ JACK OHM(1)
}

0 . Introduction. This investigation arose from the recent discovery that an integral domain $D$ may be integrally closed without the power series ring $D[[x]]$ being integrally closed, which is a consequence (by considering, for example, a valuation ring of rank $>1$ ) of the following theorem:

0.1 Theorem ([1-a, p. 76, Exercise 27], [17]). Let $D$ be an integrally closed domain. Then $D[[x]]$ integrally closed implies $\bigcap_{i=0}^{\infty} a^{i} D=0$ for every nonunit $a \in D\left({ }^{2}\right)$.

We originally set out to determine if the converse to this theorem is valid; and we show in $\$ 2$ that it is not. Proceeding from this point, we were led to a consideration of the following statements:

(a) $D$ is an intersection of rank 1 valuation rings,

(b) $D$ is completely integrally closed( $\left.{ }^{3}\right)$,

(c) $D[[x]]$ is integrally closed,

(d) $D$ is integrally closed, and $\bigcap_{i=0}^{\infty} a^{i} D=0$ for every nonunit $a \in D$.

(e) $D$ is integrally closed and every nonunit of $D$ is in a minimal prime ideal. All these statements are known to be valid for a arbitrary noetherian integrally closed domain $D$ (apply 0.2 below and [19, p. 42, Corollary 3]). Moreover, it is easily seen (Corollary 1.9) that they are all equivalent for a $D$ which is a finite intersection of valuation rings. In general,

Received by the editors June 29, 1965.

(1) During the writing of this paper the author was supported by the Wisconsin Alumni Research Foundation and by the National Science Foundation (grant GP-4074). Our interest in the subject arose out of conversations (with P. Samuel and A. Seidenberg) at the Woods Hole algebraic geometry conference, during which we received support from the American Mathematical Society.

(2) The version of this theorem given in [17] requires that $D$ contain a field, while that of [1-a] is valid for an arbitrary $D$. Seidenberg suggests the following procedure to remove the requirement from the last two theorems of [17] that the domain $\mathcal{O}$ (in the notation of [17]) should contain a field: If $\mathcal{O}$ is of characteristic $p \neq 0$, then $\mathcal{O}$ contains a field and the proof remains unchanged. If char $\mathcal{O}=0$, take $n=2$ and apply the same reasoning to $b^{n}+4 b^{n-2} t$ as was previously applied to $b^{n}+b^{n-2} t$.

(3) An element $z \in K$ is called quasi-integral over $D$ if there exists $d \neq 0 \in D$ such that $d z^{i} \in D$ for all $i>0$. $D$ is called completely integrally closed if it contains all such $z$. For these definitions we refer the reader to Bourbaki [1-a]. 
0.2 ThEOREM. (a) $\Rightarrow(\mathrm{b}) \Rightarrow(\mathrm{c}) \Rightarrow(\mathrm{d})$.

Proof. $(\mathrm{a}) \Rightarrow(\mathrm{b})$ : By $[8$, p.170, Theorem 8], every rank 1 valuation ring is completely integrally closed; so if $D$ is an intersection of rank 1 valuation rings, then $D$ is also completely integrally closed.

(b) $\Rightarrow$ (c): [1-a, p. 20, Proposition 14], or [17].

(c) $\Rightarrow(d)$ : Theorem 0.1 . q.e.d.

Thus, our first concern is with producing counterexamples to show

$$
\text { (d) } \Rightarrow \text { (c) } \Rightarrow \text {.(b) } \Rightarrow \text { (a). }
$$

As for (e), it is easy to show that $(e) \Rightarrow(d)$ (Corollary 1.4). The example constructed in $\$ 2$ shows that (e) $\Rightarrow(\mathrm{c})$, and finally in $\$ 5$ we construct an example which shows that $(a) \nRightarrow(e)$. This last example also answers a question raised by Ribenboim in [14]. We have thus succeeded in determining all possible implications between the statements (a)-(e).

Our notation and terminology adhere to that of Zariski-Samuel [18], [19]. Throughout the paper we use $D$ to denote an integral domain with quotient field $K, D^{\prime}$ to denote its integral closure, and $D[[x]]$ to denote the ring of formal power series over $D$ in a single indeterminate $x$.

1. Properties of $\bigcap_{i=0}^{\infty} a^{i} D$. Let $a \neq 0$ be a nonunit of $D$. For any valuation ring $D_{v}$ such that $D \subset D_{v} \subset K$, let $P_{v}=\bigcap_{i=0}^{\infty} a^{i} D_{v} . P_{v}$ is a prime ideal of $D_{v}$ (see, for example, [3, Lemma 2.10]). Let $V$ be the set of all valuations $v$ of $K$ such that $D_{v} \supset D$.

1.1 Proposition. $\bigcap_{i=0}^{\infty} a^{i} D^{\prime}=\bigcap_{v \in V} P_{v}=\bigcap_{v \in V}\left(P_{v} \cap D^{\prime}\right)$.

Proof.

$$
\begin{aligned}
\bigcap_{v \in V} P_{v} & =\bigcap_{v \in V}\left(\bigcap_{i=0}^{\infty} a^{i} D_{v}\right) \\
& =\bigcap_{i=0}^{\infty}\left(\bigcap_{v \in V} a^{i} D_{v}\right) \\
& =\bigcap_{i=0}^{\infty} a^{i} D^{\prime}, \text { since } D^{\prime}=\bigcap_{v \in V} D_{v} .
\end{aligned}
$$

The second equality follows from the fact that $\bigcap_{v \in V} P_{v} \subset D^{\prime}$.

1.2 CoRollary. $\bigcap_{i=0}^{\infty} a^{i} D^{\prime}$ is its own radical.

Let now $P \neq 0$ be a prime ideal of $D$. We shall use the notation $F(P)$ to denote a first prime below $P$, i.e. $F(P)$ is a prime ideal of $D$ such that $F(P)<P$ and such that there exists no prime ideal property between $F(P)$ and $P$. By Zorn's lemma, 
there always exists such an $F(P)$ when $P$ is a minimal prime divisor of a principal ideal, but in general $F(P)$ is not uniquely determined by $P$.

1.3 TheOREM. Let $P$ be a minimal prime divisor of $a D$, and let $F(P)$ be a first prime below $P$. Then there exists a $v \in v$ such that $F(P)=P_{v} \cap D$. Moreover, if $D$ is a Prufer domain( $\left.{ }^{4}\right)$, then $F(P)$ is unique and $F(P)=P_{v} \cap D$ for any $v \in V$ such that $P D_{v} \neq D_{v}$.

Proof. There exists a valuation ring $D_{v}$ of $K$ containing $D$ and having prime ideals which lie over $F(P)$ and $P\left[11\right.$, p. 37, (11.9)]. If $P^{\prime}$ is the intersection of the prime ideals lying over $P$ and $F^{\prime}$ the union of the prime ideals lying over $F(P)$, then $F^{\prime}$ lies over $F(P)$ and is a first prime below $P^{\prime}$ in $D_{v}$. Therefore $a \in P^{\prime}$ and $a \notin F^{\prime}$ implies $F^{\prime}=\bigcap_{i=0}^{\infty} a^{i} D_{v}=P_{v}$. Thus, $P_{v} \cap D=F(P)$.

Now assume $D$ is a Prufer domain. Then the prime ideals of $D$ which are contained in a given prime ideal are linearly ordered, so $F(P)$ is uniquely characterized as the union of the primes strictly contained in $P$. For any $v \in V$ such that $P D_{v} \neq D_{v}$, since $D_{v}$ is a quotient ring of $D$, the fact that $P_{v}$ is a first prime below $P D_{v}$ implies $P_{v} \cap D$ is a first prime below $P$. Thus, $F(P)=P_{v} \cap D$.

1.4 Corollary. If $a$ is in a minimal prime ideal of $D$, then $\bigcap_{i=0}^{\infty} a^{i} D=0\left({ }^{5}\right)$.

Proof. By 1.3 there exists a $v \in V$ such that $P_{v} \cap D=0$. But $\bigcap_{i=0}^{\infty} a^{i} D \subset P_{v} \cap D$ by 1.1 .

1.5 Corollary. If $D$ is a Prufer domain and $\left\{P_{\alpha}\right\}$ is the set of minimal prime divisors of $a D$, then $\bigcap_{i=0}^{\infty} a^{i} D=\bigcap F\left(P_{\alpha}\right)$.

Proof. $D=D^{\prime}$ for a Prufer domain, so by $1.1 \bigcap_{i=0}^{\infty} a^{i} D=\bigcap_{v \in V}\left(P_{v} \cap D\right)$. By 1.3, for any $P_{\alpha}, F\left(P_{\alpha}\right)=P_{v} \cap D$ for some $v \in V$. On the other hand, if $P_{v} \neq D_{v}$, then $a D_{v} \neq D_{v}$ and hence there exists a $P_{\alpha}$ such that $P_{\alpha} D_{v} \neq D_{v}$. Therefore again by $1.3, P_{v} \cap D=F\left(P_{\alpha}\right)$. Thus, $\bigcap F\left(P_{\alpha}\right)=\bigcap_{v \in V}\left(P_{v} \cap D\right)$.

1.6 Corollary. Let $D$ be a Prufer domain, and suppose $a \neq 0$ is a nonunit of $D$ which is in only finitely many maximal ideals. Then $\bigcap_{i=0}^{\infty} a^{i} D=0$ if and only if $a$ is in a minimal prime ideal of $D$.

Proof. Since the prime ideals which are contained in a given maximal ideal are linearly ordered, $a D$ has only finitely many minimal prime divisors $\left\{P_{\alpha}\right\}$. By 1.5, $\bigcap_{i=0}^{\infty} a^{i} D=\bigcap F\left(P_{\alpha}\right)=\pi F\left(P_{\alpha}\right)$. But $\pi F\left(P_{\alpha}\right)=0$ if and only if $F\left(P_{\alpha}\right)=0$ for some $\alpha$, and $F\left(P_{\alpha}\right)=0$ if and only if $P_{\alpha}$ is a minimal prime ideal. q.e.d.

1.4 shows that $(e) \Rightarrow(d)$. Finite intersections of valuation rings are exactly the Prufer domains with only a finite number of maximal ideals (apply $[11$, p. 38,

(4) $D$ is a Prufer domain if $D_{P}$ is a valuation ring for every proper prime ideal $P$ of $D$. For equivalent definitions see [1-b, p. 93, Exercise 12] or [3, Theorem 2.2].

(s) This corollary could also be obtained by applying [13, Lemma 1, p. 89]. 
(11.11)]), so the above Corollary 1.6 asserts that $(e) \Leftrightarrow(d)$ when $D$ is a finite intersection of valuation rings. In general, $(d) \Rightarrow(e)$, as we shall eventually see.

(1.7) A subclass of the set of Prufer domains is formed by the integral domains with $Q R$ ([2, p. 99, Theorem 2.5]). $D$ is said to have the $Q R$-property if every ring $D_{1}$ such that $D \subset D_{1} \subset K$ is a quotient ring of $D$ with respect to a multiplicative system. Included among the domains with $Q R$ are the domains with the property that every finitely generated ideal is principal [2, p. 99, Corollary $2.4]$, and thus in particular the finite intersections of valuation rings.

\subsection{Proposition. If $D$ is a domain with $Q R$, then $(\mathrm{a}) \Leftrightarrow(\mathrm{e})$.}

Proof. By [2, p. 98, Proposition 1.2], $\bigcap D_{P}=D_{U P}\left({ }^{6}\right)$, where $P$ range; over the minimal prime ideals of $D$; and each $D_{P}$ is a rank 1 valuation ring, by [2, p. 99, Theorem 2.5]. Therefore, if every nonunit of $D$ is in a minimal prime ideal, then $D=D_{U P}=\bigcap D_{P}$; and thus $D$ is an intersection of rank 1 valuation rings. Conversely, if $D=\bigcap D_{v}, D_{v}$ a rank 1 valuation ring, then each $D_{v}=D_{P}$ for some minimal prime ideal $P$ of $D$; and thus, as before $D=\bigcap D_{P}=D_{U P}$, where $P$ ranges over the minimal prime ideals. But $D=D_{U P}$ implies every nonunit of $D$ is in a minimal prime. q.e.d.

1.9 Corollary. Let $D$ be a finite intersection of valuation rings. Then (a), (b), (c), (d), (e) are equivalent for $D$.

Proof. By 0.2 , we have seen that quite generally (a) $\Rightarrow(\mathrm{b}) \Rightarrow(\mathrm{c}) \Rightarrow(\mathrm{d})$. Moreover, (e) $\Leftrightarrow($ a) for $D$ a finite intersection of valuation rings by 1.8 . Therefore we need only observe that $(\mathrm{d}) \Rightarrow(\mathrm{e})$, by 1.6 . q.e.d.

A valuation ring is completely integrally closed if and only if it has rank 1 $[8$, p. 170 , Theorem 8$]$; so in view of $[11$, p. $38,(11.11)]$ and the above corollary, (a)-(e) are equivalent to the assertion that $D$ is 1 -dimensional, when $D$ is a finite intersection of valuation rings. From this one might be led to conjecture that a Prufer domain which is completely integrally closed is also 1-dim (the converse being immediate). That this is false follows from the example of the ring of entire functions, [1-a, p. 71, Exercise 12] or [4], [5]( $\left.{ }^{7}\right)$. (Note that the ring of entire functions even has the $Q R$-property, since every finitely generated ideal is principal $[5$, p. 712$]$.)

2. An example to show $(d) \nRightarrow(c)$. We construct now an example of a domain for which (e) $\Rightarrow$ (c). Since (e) $\Rightarrow$ (d) by 1.4, this then also shows that (d) $\Rightarrow$ (c).

2.1 EXAMPLE. Let $k$ be a field of characteristic $\neq 2$, let $d, a_{0}, a_{1}, \cdots$ be indeterminates over $k$, and let $K=k\left(d, a_{0}, a_{1}, \cdots\right)$. If $w=d^{-1} \sum_{i=0}^{\infty} a_{i} x^{i}$, then $w^{2}=b_{0}^{2}+\sum_{i=1}^{\infty} b_{i} x^{i}$, where $b_{0}=a_{0} d^{-1}, \quad b_{n}=d^{-2} \sum_{i=0}^{\infty} a_{i} a_{n-i}, \quad n \geqq 1$. Let $D=k\left[d, a_{0}, a_{1}, \cdots ; b_{0}, b_{1}, \cdots\right]$, and let $D^{\prime}=$ integral closure of $D . w^{2} \in D^{\prime}[[x]]$,

(6) $D_{U P}$ denotes the quotient ring of $D$ with respect to the multiplicative system $D-\bigcup P$.

(7) This remark is the result of a conversation with I. Kaplansky. 
and hence $w$ is integral over $D^{\prime}[[x]]$. Also $d w=\sum_{i=0}^{\infty} a_{i} x^{i} \in D^{\prime}[[x]]$, so $w$ is in the quotient field of $D^{\prime}[[x]]$. By the following lemma, $w \notin D^{\prime}[[x]]$.

2.2 LEMMA. $a_{1} d^{-1} \notin D^{\prime}$.

Proof. The natural grading of $k\left[d, a_{0}, a_{1}, \cdots\right]$ induces a grading on a subring of $K$ : $\Sigma_{-\infty}^{\infty} K_{i}$ (see [19, p. 157]). Since $D$ is generated by homogeneous elements of $K$, $D=\sum_{i=0}^{\infty} D_{i}$, where $D_{i}=D \cap K_{i}\left[16\right.$, p. 11, Lemma 3.2]. If now $D_{0}^{\prime}=D^{\prime} \cap K_{0}$, then $D_{0}^{\prime}$ is the integral closure of $D_{0}$ in $K[19$, p. 159, Remark]. But $D_{0}=k\left[b_{0}, b_{1}, \cdots\right]$, and since characteristic of $k \neq 2$, this is a polynomial ring over $k$ in $b_{0}, b_{1}, \cdots$. Therefore $D_{0}$ is a UFD.

If $a_{1} d^{-1} \in D^{\prime}$, then $a_{1} d^{-1} \in D_{0}^{\prime}$ and hence is integral over $D_{0}$. But $a_{1} d^{-1}=b_{1}\left(2 b_{0}\right)^{-1}$, so $a_{1} d^{-1}$ is in the quotient field of $D_{0}$ and therefore in $D_{0}$, because a UFD is integrally closed. Thus, $2 b_{0}\left(a_{1} d^{-1}\right)=b_{1}$ in $D_{0}$, a contradiction to the fact that $b_{0}, b_{1}$ are irreducible elements of $D_{0}$. q.e.d.

We have thus shown that $D^{\prime}[[x]]$ is not integrally closed. The next lemma shows that (e) holds in $D^{\prime}$.

2.3 Lemma. Every nonunit $z$ of $D^{\prime}$ is in a minimal prime ideal of $D^{\prime}$.

Proof. We consider two cases:

(i) $z \neq u d^{i}$, where $u$ is a unit of $D^{\prime}$ and $i \geqq 0$. Let $R=k\left[d, a_{0}, a_{1}, \cdots\right]$, and let $M$ be the multiplicative system in $R$ consisting of all powers of $d: M=\left\{d^{i}\right\}$, $i \geqq 1$. Then $R \subset D \subset R_{M}$. $R$ is integrally closed implies $R_{M}$ is also integrally closed [18, p. 261], so $D^{\prime} \subset R_{M}$. Therefore $z$ is a nonunit of $D^{\prime}$ implies $d^{i} z$ is a nonunit of $R$ for some $i$. Noting that $R$ is a UFD, since $z \notin M$ we can choose an irreducible factor $y$ of $d^{i} z$ such that $y$ is relatively prime to $d$. Then $y R=P$ is a minimal prime ideal of $R$ such that $P \cap M=\varnothing$. Therefore $P^{*}=P R_{M}$ is also a minimal prime ideal of $R_{M}$. Since $R \subset D^{\prime} \subset R_{M}, D_{M}^{\prime}=R_{M}$. Therefore by the correspondence between prime ideals of $D^{\prime}$ and $D_{M}^{\prime}, P^{*} \cap D^{\prime}$ is also a minimal prime ideal of $D^{\prime}$; and thus $z \in P^{*} \cap D^{\prime}$ shows that $z$ is in a minimal prime ideal of $D^{\prime}$.

(ii) $z=u d^{i}$ for some unit $u$ of $D^{\prime}$ and some $i \geqq 1$. It is sufficient to see that $d$ is in a minimal prime ideal of $D^{\prime}$. Let $S=k\left[b_{0}, b_{1}, \cdots\right]$, and let $N$ be the multiplicative system in $S$ consisting of powers of $b_{0}$. Then $a_{n} d^{-1} \in S_{N}$ follows by induction on $n: n=0 . a_{0} d^{-1}=b_{0} \in S_{N}$. Assuming $a_{i} d^{-1} \in S_{N}$ for $i<n$, then

$$
b_{n}=d^{-2} \sum_{i=0}^{n} a_{i} a_{n-i},
$$

for $n \geqq 1$, implies $2 a_{n} a_{0} d^{-2}=b_{n}-d^{-2} \sum_{i=1}^{n-1} a_{i} a_{n-i} \in S_{N}$ by induction hypothesis. Therefore $2\left(a_{n} d^{-1}\right) b_{0} \in S_{N}$, so $a_{n} d^{-1} \in S_{N}$.

Thus, if $T=k\left[d, b_{0}, b_{1}, \cdots\right]$, then $D \subset T_{N}$. Since $T$ is a UFD and hence integrally closed, $T_{N}$ is also integrally closed; so $D^{\prime} \subset T_{N}$, and then $D_{N}^{\prime}=T_{N}$. If now $P=d T$, then $P$ is a minimal prime ideal of $T$ such that $P \cap N=\varnothing$. 
Therefore $P^{*}=P T_{N}$ is also a minimal prime ideal of $T_{N}$; and hence $P^{*} \cap D^{\prime}$ is a minimal prime ideal of $D^{\prime}$, because $T_{N}$ is a quotient ring of $D^{\prime}$. Now observe that $d \in P^{*} \cap D^{\prime}$. q.e.d.

We have thus proved that $D^{\prime}$ has the following properties:

(1) $D^{\prime}$ is integrally closed,

(2) $D^{\prime}[[x]]$ is not integrally closed,

(3) every nonunit of $D^{\prime}$ is in a minimal prime ideal. Thus, $D^{\prime}$ provides the required example.

Note that we have shown that $D^{\prime}[[x]]$ is not even integrally closed in the quotient ring $D^{\prime}[[x]]_{D}$. Thus, one problem is to find sufficient conditions on $D$ in order for $D[[x]]$ to be integrally closed in $D[[x]]_{D}$. Another problem is the question of whether $D$ integrally closed implies $D[[x]]_{D}$ is integrally closed. Part of the difficulty here seems to be the obscure nature of the quotient field $\Sigma$ of $D[[x]]$. For instance, it can happen that $\Sigma$ does not even contain $K[[x]]$, as shown by the following example $\left(^{8}\right)$.

2.4 Example. Let $D=k\left[a, a_{0}, a_{1}, \cdots\right]$ be a polynomial ring over $k$, and let $y=\sum_{i=0}^{\infty} a_{i}(x / a)^{i}$. Then $y \in K[[x]]$.

Claim: $y \notin \Sigma$. For, if $y \in \Sigma$, then $y=\sum_{i=0}^{\infty} c_{i} x^{i} / \sum_{i=0}^{\infty} b_{i} x^{i}, c_{i}, b_{i} \in D$. Then by equating coefficients,

$$
\left(a_{0} b_{n}-c_{n}\right) a^{n}+\left(a_{1} b_{n-1}\right) a^{n-1}+\cdots+\left(a_{n-1} b_{1}\right) a+a_{n} b_{0}=0 \quad \text { for } n \geqq 1 .
$$

Also, $a_{0} b_{0}=c_{0}$ implies $c_{0}=0$ when $b_{0}=0$. Therefore we may assume $b_{0} \neq 0$.

LeMmA. $a^{i-j} \mid b_{j}, j=0, \cdots, i-1$ implies $a^{i-j+1} \mid b_{j}, j=0, \cdots, i$.

Proof. By hypothesis, $b_{j}=a^{i-j} b_{j}^{\prime}, b_{j}^{\prime} \in D, j=0, \cdots, i-1$; so by $\left(^{*}\right)$ we have for $n>i$ :

$$
a^{i}\left(a_{n} b_{0}^{\prime}+a_{n-1} b_{1}^{\prime}+\cdots+a_{n-i+1} b_{i-1}^{\prime}\right)+\left(a_{n-i} b_{i}\right) a^{i}+a^{i+1}\{\quad\}=0 .
$$

Therefore

$$
a_{n} b_{0}^{\prime}+a_{n-1} b_{1}^{\prime}+\cdots+a_{n-i+1} b_{i-1}^{\prime}+a_{n-i} b_{i} \equiv 0(a D) .
$$

Choosing $n$ so large that $b_{0}^{\prime}, \cdots, b_{i-1}^{\prime}, b_{i}$ are polynomials which involve only $a_{l}$ with $l<n-i$, we then have $b_{0}^{\prime} \equiv b_{1}^{\prime} \equiv \cdots \equiv b_{i-1}^{\prime} \equiv b_{i} \equiv 0(a D)$. Therefore $a$ divides $b_{0}^{\prime}, b_{1}^{\prime}, \cdots, b_{i-1}^{\prime}, b_{i}$, which proves the lemma. q.e.d.

Continuing with the proof of $y \notin \Sigma$, observe that $\left(a_{0} b_{1}-c_{1}\right) a+a_{1} b_{0}=0$ implies $a \mid b_{0}$, since $a$ does not divide $a_{1}$ in the UFD $D$. Thus, by the lemma used

(8) As the referee has pointed out, Example 2.4 is complicated mainly in order to illustrate the remark at the end of 2.5 . If one merely wants to show that $K[[x]] \notin \Sigma$, it suffices to consider $\sum_{i=0}^{\infty} a_{i}^{-1} x^{i}$. We would also like to mention that an example closely resembling our considerations in 2.4 and 2.5 can be found in [15]. 
inductively on $i, a^{i-j} \mid b_{j}, j=0, \cdots, i-1$, for all $i \geqq 1$. In particular then, $a^{i} \mid b_{0}$ for all $i \geqq 1$.

Therefore $b_{0}=0$, which is a contradiction to our previous assumption. Therefore $y \notin \Sigma$. q.e.d.

The above example actually shows that $D[[x / a]] \nsubseteq \Sigma$. Observe also that $a /(a-x)=\sum_{i=0}^{\infty}(x / a)^{i} \in \Sigma$ for all $a \neq 0$. But $a /(a-x) \in D[[x]]_{D}$ if and only if $\bigcap_{i=0}^{\infty} a^{i} D \neq 0$. Therefore if $D[[x]]$ is integrally closed, then

$$
D[[x / a]] \cap \Sigma \notin D[[x]]_{D} \text {. }
$$

In this connection, the following might also be of some interest:

2.5 Proposition. Let $\Sigma$ be the quotient field of $D[[x]]$. Then

$$
K[[x]] \cap \Sigma \subset \bigcup a^{-1} D[[x / a]], \quad a \neq 0 \text { in } D .
$$

Proof. If $y \in K[[x]] \cap \Sigma$, then $y=\sum_{i=0}^{\infty} a_{i} x^{i}, a_{i} \in K$; and

$$
y=\sum_{i=0}^{\infty} c_{i} x^{i} / \sum_{i=0}^{\infty} b_{i} x^{i}, \quad b_{i}, c_{i} \in D .
$$

Since $a_{0} b_{0}=c_{0}, b_{0}=0$ implies $c_{0}=0$; so we may assume $b_{0} \neq 0$. Equating coefficients,

$$
a_{n}=b_{0}^{-1}\left(c_{n}-\sum_{i=1}^{n} b_{i} a_{n-i}\right), \quad n \geqq 1 .
$$

Then by induction on $n$, this formula yields $a_{n}=d_{n} / b_{0}^{n+1}$ for some $d_{n} \in D$. Therefore $y=b_{0}^{-1} \sum_{i=0}^{\infty} d_{i}\left(x / b_{0}\right)^{i} \in b_{0}^{-1} D\left[\left[x / b_{0}\right]\right]$. q.e.d.

Example 2.4 shows that the containment of the above proposition may actually be proper containment.

3. Overrings and (c) $\Rightarrow($ b). Let $D$ be as before a domain with quotient field $K$, and let $R$ be a domain such that $D \subset R$. Let $C(R)=[D: R]_{D}=\{d \in D \mid d R \subset D\}$. Then $C(R)$ is an ideal in $D$ and also an ideal in $R$, and it contains any other such ideal.

3.1 Proposition. (i) $\bigcap_{i=0}^{\infty} a^{i} D=C(R)$, where $a \neq 0$ is any nonunit of $D$ and $R=D[1 / a]$.

(ii) $D$ is completely integrally closed if and only if $C(R)=0$ for every ring $R$ such that $D<R \subset K$.

Proof. (i) If $R=D[1 / a], c \in C(R)$ if and only if $c(1 / a)^{i} \in D$ for all $i$, i.e. if and only if $c \in \bigcap_{i=0}^{\infty} a^{i} D$.

(ii) If $C(R) \neq 0$ for some $R>D$, then there exists an $r \in R, \notin D$ and $c \in D$ such that $c r^{i} \in D$ for all $i$. Therefore $r$ is quasi-integral over $D$ and yet not in $D$, so $D$ is not completely integrally closed. Conversely, if $r \in K$ is quasi-integral over 
$D$ and $r \notin D$, then there exists $c \neq 0$ in $D$ such that $c r^{i} \in D$ for all $i$. Therefore if $R=D[r]$, then $c \in C(R)$; and hence $C(R) \neq 0$. q.e.d.

Thus, properties such as $D$ completely integrally closed and $\bigcap_{i=0}^{\infty} a a^{i} D=0$ for every nonunit $a \in D$ are reflected in $C(R)$ being 0 for a certain class of overrings of $D$. Question: Is there a class of overrings of $D$ for which $C(R)=0$ is equivalent to the assertion that $D[[x]]$ is integrally closed?

We turn next to the study of a special situation which is connected with the counterexample that shows $(c) \nRightarrow(b)$.

3.2 Theorem. Let $D^{*}=\{r \in R \mid r$ is integral over $D\}$, and let

$$
D[[x]]^{*}=\{z \in R[[x]] \mid z \text { is integral over } D[[x]]\} \text {. }
$$

If $D=U+C(R)\left({ }^{9}\right)$, where $U$ consists of the units of $D$ and 0 , then

$$
D[[x]]^{*} \subset D^{*}[[x]]
$$

Proof. Let $z \in D[[x]]^{*}$ and suppose $z \notin D^{*}[[x]]$. Then $z=\sum_{i=0}^{\infty} a_{i} x^{i}, a \in R$. Let $a_{t}$ be the first coefficient of $z$ which is not in $D^{*}$, and let $w=z-\sum_{i=0}^{t-1} a_{i} x^{i}$. Then since $D[[x]]^{*}$ is a ring containing $D^{*}[x], w \in D[[x]]^{*}$ also. Therefore there exist $y_{1}, \cdots, y_{n} \in D[[x]]$ such that $w^{n}+y_{1} w^{n-1}+\cdots+y_{n}=0$. We can write $y_{i}=y_{i}^{\prime}+c_{i}, i=1, \cdots, n-1$, where $y_{i}^{\prime} \in U[[x]], c_{i} \in C(R)[[x]]$; and then

$$
w^{n}+y_{1}^{\prime} w^{n-1}+\cdots+y_{n-1}^{\prime} w+\left(c_{1} w^{n-1}+\cdots+c_{n-1} w+y_{n}\right)=0 .
$$

Letting $y_{n}^{\prime}=c_{1} w^{n-1}+\cdots+c_{n-1} w+y_{n}$, then $y_{n}^{\prime} \in D[[x]]$ also, since $c_{i} \in C(R)[[x]]$. Consider now the summands of $\left({ }^{* *}\right)$ having minimal subdegree $m$ in $x$. Since the subdegree of $y_{n}^{\prime}$ is $\geqq$ the minimum of the subdegrees of the other summands of $(* * *)$, at least one such summand involves $w$. Dividing $(* * *)$ by $x^{m}$ and equating to 0 the resulting constant term, we get a relation

$$
b_{l} a_{t}^{n-l}+b_{l+1} a_{t}^{n-l-1}+\cdots+b_{n}=0, \quad b_{i} \in D, \quad b_{l} \neq 0 \quad \text { and } \quad b_{l} \in U .
$$

Therefore $b_{l}^{-1} \in D$, and hence $a_{t}^{n-l}+\left(b_{l+1} b_{l}^{-1}\right) a_{t}^{n-l-1}+\cdots+\left(b_{n} b_{l}^{-1}\right)=0$ is an equation of integral dependence for $a_{t}$ with respect to $D$. Thus, $a_{t} \in D^{*}$, a contradiction to our choice of $t$.

3.3 Proposition. Suppose $D \subset R$ and $R=R_{0} \oplus C(R)\left({ }^{10}\right)$ where $R_{0}$ is a subring of $R$. Then $D=D_{0} \oplus C(R)$, where $D_{0}=D \cap R_{0}$; and if $D_{0}$ is integrally closed in $R_{0}$, then $D$ is integrally closed in $R$.

Proof. The first assertion is immediate. Suppose then $r \in R$ is integral over $D$.

(9) Note that $U$ need not be a ring. The notation $U+C(R)$ denotes the set of all $u+c$, $u \in U, c \in C(R)$.

(10) $\oplus$ denotes direct sum of groups. 
$r=r_{0}+c, r_{0} \in R_{0}, c \in C(R)$; so $r_{0}=r-c$ is also integral over $D$. Thereforc there exist $a_{1}, \cdots, a_{n} \in D$ such that $r_{0}^{n}+a_{1} r_{0}^{n-1}+\cdots+a_{n}=0$. We can write $a_{i}=a_{i}^{\prime}+c_{i}, a_{i}^{\prime} \in D_{0}, c_{i} \in C(R)$. Then $\left(r_{0}^{n}+a_{1}^{\prime} r_{0}^{n-1}+\cdots+a_{n}^{\prime}\right)+\left(c_{1} r_{0}^{n-1}+\cdots+c_{n}\right)=0$. Since $\left(c_{1} r_{0}^{n-1}+\cdots+c_{n}\right) \in C(R)$, this implies $r_{0}^{n}+a_{1}^{\prime} r_{0}^{n-1}+\cdots+a_{n}^{\prime}=0$. Therefore $r_{0}$ is integral over $D_{0}$ and hence $\in D_{0}$. Then $r \in D$.

3.4 Corollary. Suppose $D \subset R$ and $R=k \oplus C(R)$, where $k$ is a field contained in $R$. Let $D_{0}=D \cap k$. If $D_{0}[[x]]$ is integrally closed in $k[[x]]$ and if $\bigcap_{i=0}^{\infty} a_{i} D=0$ for every $a \in D_{0}$ such that $a$ is a nonunit of $D$, then $D[[x]]$ is integrally closed in $R[[x]]$.

Proof. By 3.3, $D=D_{0} \oplus C(R)$ and $D$ is integrally closed in $R$. Moreover, if $a \neq 0 \in D_{0}$, then $1 / a \in R$ and hence $(1 / a)^{i} c \in D$ for every $i \geqq 0$ and every $c \in C(R)$. Therefore $C(R) \subset \bigcap_{i=0}^{\infty} a^{i} D$. If $\bigcap_{i=0}^{\infty} a^{i} D=0$, then $C(R)=0$ and $D=D_{0}, R=k$; so the corollary is immediate. If $\bigcap_{i=0}^{\infty} a^{i} D \neq 0$, then by hypothesis $a$ is a unit in $D$. Thus, in this case the nonzero elements of $D_{0}$ are units of $D$, so we can apply 3.2 to conclude that $D[[x]]$ is integrally closed in $R[[x]]$. q.e.d.

In [10, pp. 670-671], Krull studies the following example: Let $K=k(x, y)$ be the field of rational functions in two indeterminates $x, y$ over a field $k$. There exists a discrete rank 1 valuation $v$ of $K$ such that $v(x)=1, v(y)=0$ and such that if $R$ is the valuation ring of $v$, then $R=k(y) \oplus M$, where $M$ is the maximal ideal of $R$. Let $D=k \oplus M$. Then Krull shows that $D$ is a 1-dim quasi-local ring with maximal ideal $M$, and that $D$ is integrally closed but not completely integrally closed. Moreover, by $3.2 k[[x]]$ is integrally closed in $k(y)[[x]]$, so by $3.4 D[[x]]$ is integrally closed in $R[[x]]$. But $R[[x]]$ is integrally closed (apply 1.9 ), so therefore $D[[x]]$ is also integrally closed. Thus, this is an example of a domain $D$ for which (c) $\Rightarrow\left(\right.$ b) $\left({ }^{11}\right)$.

4. (b) $\Rightarrow$ (a). In [12], Nakayama constructs an example of a completely integrally closed domain $D$ such that $D$ is not contained in any rank 1 valuation ring of its quotient field $K$. He also points out how one can get from such a $D$ to one which is completely integrally closed and such that not every nonunit is in a minimal prime ideal. Thus, in particular, his examples show that (b) $\Rightarrow$ (a) and (b) $\Rightarrow$ (e). The procedure used by Nakayama is to first construct a lattice ordered group $G$ with the correct properties and then to show that there exists a domain $D$ such that $G$ is the group of divisibility of $K$ with respect to $D$ (see Jaffard [6, p. 5], for the definitions). Instead of using Nakayama's construction, one can in general prove the existence of a $D$ having a given lattice group as its associated group of divisibility by employing Jaffard [6,p. 78, Theorem 3$]$. An examination of the proof of this theorem shows that the resulting $D$ is, in fact, a domain with the property that every finitely generated ideal is principal; so in particular $D$ has the $Q R$-property (see 1.7). This construction is related to Krull's construction of the

(11) We are indebted to P. Samuel for pointing out this example. 
Kronecker function ring [9, pp. 558-561], and may be regarded as a special case of it. (We hope to enlarge on this statement in a future paper.)

The example which we give in the next section shows that (a) $\Rightarrow$ (e). It also proves that $(\mathrm{b}) \nRightarrow(\mathrm{e})$ and hence provides an alternative approach to Nakayama's second counterexample. Note that since (a) $\Leftrightarrow$ (e) for a domain with $Q R$ (by 1.8 ), the above method of passing from a lattice group with the correct properties to a domain $D$ via Jaffard's theorem will not produce an example for which (a) $\nRightarrow$ (e).

5. (a) $\nRightarrow(e)$. In this section we construct an example which shows that (a) $\Rightarrow$ (e). To fix the notation, $l \epsilon_{\imath} D, R, R_{v}$ be domains having quotient field $K$ and such that $R_{v}$ is the valuation ring of a valuation $v$ of $K$. Furthermore, assume that $D=R \cap R_{v}$ and that $R_{v}$ is irredundant in this intersection, i.e. $R \notin R_{v}$. Let $M_{v}$ be the maximal ideal of $R_{v}$ and let $M=M_{v} \cap D$.

5.1 THEOREM. If the value group of $v$ is contained in the rational numbers, then $M$ is a minimal prime ideal of $D$.

Proof. Let $d \neq 0 \in D$, and assume $P$ is a prime ideal of $D$ such that $P<M$. Since $R \notin R_{v}$, there exists $z \in R, \notin R_{v}$. Therefore $v(z)<0$, and hence there exists integers $m \geqq 0, n>0$ such that $v(d) /-v(z)=m / n$. Therefore $n v(d)+m v(z)=0$, or $v\left(d^{n} z^{m}\right)=0$. This implies $d^{n} z^{m} \in D, \notin M$. Now choose $y \in M, \notin P$. Then $v(y)>0$, so there exists an integer $q>0$ such that $q v(y)>v(d)$. Therefore

$$
v\left(d^{n} z^{m} y^{q}\right)=q v(y)>v(d)
$$

and hence $v\left(d^{n-1} z^{m} y^{q}\right)>0$, so $d^{n-1} z^{m} y^{q} \in D$. But $d\left(d^{n-1} z^{m} y^{q}\right) \notin P$ since $d^{n} z^{m} \notin M$ and $y \notin P$. Therefore $d \notin P$, and hence $P=0$.

It remains to see that $M \neq 0$. If $M=0$, then $v$ is 0 on $D$ and consequently also on $K$. But then $R_{v}=K \supset R$, a contradiction to our assumption that $R_{v}$ is irredundant.

5.2 COROLlARY. If a domain $D$ is an irredundant intersection of rational, rank one valuation rings, then each of these valuations is centered on a minimal prime ideal of $D$, and consequently every nonunit of $D$ is in a minimal prime ideal of $D$.

Thus, in searching for an example to show (a) $\Rightarrow(e)$, at least in the case of an irredundant intersection, one must look for a domain whose representation as an intersection of rank one valuation rings involves at least one nonrational valuation. We now proceed to the construction of such an example.

5.3. EXAmPLe. Let $k=$ rational numbers, $J=$ integers, and fix a prime integer $p$. $J_{p}$ will denote the quotient ring of $J$ with respect to the prime ideal $p J$. Let $x$ be an indeterminate, and let $v$ denote the extension of the $p$-adic valuation of $k$ to $k(x)$ obtained by defining $v\left(a_{0}+a_{1} x+\cdots+a_{n} x^{n}\right)=\inf \left\{v\left(a_{i}\right)+i \pi\right\}, i=0, \cdots, n$. Let $R_{v}$ be the valuation ring of $v$, let $R=k[x]$, and let $D=R \cap R_{v}$. Since $R$ is a 
Dedekind domain, $R_{P}$ is a discrete, rank one valuation ring for every proper prime ideal $P$ of $R$, and $R=\bigcap R_{P}$. Thus, $D=\left(\bigcap R_{P}\right) \cap R_{v}$ is a representation of $D$ as an intersection of rank one valuation rings; and, in fact, every element of $D$ is a nonunit in at most a finite number of these valuation rings.

5.4 LEMMA. None of the valuation rings $\left\{R_{P}\right\}, R_{v}$ is redundant in the representation $D=\left(\bigcap R_{P}\right) \cap R_{v}$.

Proof. $R_{v}$ is irredundant since $1 / p \in R, \notin R_{v}$. If $Q$ is a proper prime ideal of $R$, then $Q=z R$ for some $z \neq 0 \in R$; so for sufficiently large $n, p^{n} / z \in\left(\bigcap_{P \neq Q} R_{P}\right) \cap R_{v}$ and $\notin R_{Q}$. Therefore $R_{Q}$ is irredundant. q.e.d.

If $M_{v}$ is the maximal ideal of $R_{v}$, as before let $M=M_{v} \cap D$; and similarly, for the maximal ideal $P R_{P}$ of $R_{P}$, let $P^{*}=P R_{P} \cap D$.

5.5 Corollary. For any proper prime ideal $P$ of $R, P^{*}=P \cap D$ is a minimal prime ideal of $D$.

Proof. Apply 5.4 and 5.1.

5.6 LEMMA. $\sqrt{ }(p D)=M$.

Proof. $\sqrt{ } p \in M$, so $(p D) \subset M$. Conversely, if $y \in M$, then $v(y)>0$. Therefore there exists an integer $n$ such that $v\left(y^{n} / p\right)>0$, and hence $y^{n} / p \in D$. But then $y_{n} \in p D$, so $y \in \sqrt{ }(p D)$.

5.7 LEMMA. The only proper prime ideals of $D$ are the $\left\{P^{*}\right\}$ and $M$.

Proof. Let $S$ be the multiplicative system in $J_{p}$ consisting of powers of $p$. Then $J_{p}[x] \subset D \subset R$, and $R=J_{p}[x]_{s}$. Therefore $R=D_{S}$ also, so any proper prime ideal of $D$ which does not intersect $S$ must be the contraction of a proper prime ideal of $R$ and hence must be some $P^{*}$.

Suppose then that $Q$ is a prime ideal of $D$ such that $Q \cap S \neq \varnothing$. Then $p \in Q$; and hence by 5.6, $M \subset Q$. On the other hand, if $Q>M$, then there exists $y=a_{0}+a_{1} x+\cdots+a_{n} x^{n}, \quad a_{i} \in k$, such that $v(y)=0$ and $y \in Q$. Since $v(y)=\inf \left\{v\left(a_{i}\right)+i \pi\right\}, i=0, \cdots, n$, this implies $v\left(a_{0}\right)=0$ and $v\left(a_{i}\right)+i \pi>0$ for $i>0$. Thus, $v\left(y-a_{0}\right)=\inf \left\{v\left(a_{i}\right)+i \pi\right\}>0, i=1, \cdots, n$; so $y-a_{0} \in M$. But then $y \in Q$ and $y-a_{0} \in Q$, so $a_{0} \in Q$. However, $v\left(a_{0}\right)=0$ and $a_{0} \in k$, so $a_{0}$ is a unit of $D$; and hence $Q=D$.

5.8 Lemma. $p$ is in no minimal prime ideal of $D$.

Proof. Since $\sqrt{ }(p D)=M$ (by 5.6), any prime ideal of $D$ which contains $p$ contains $M$. Let $P^{*}$ be the prime ideal $x R \cap D$.

Claim: $P^{*}<M . p \notin P^{*}$ since $p \notin x R$. On the other hand, if $y \in P^{*}$, then $y=a_{s} x^{s}+a_{s+1} x^{s+1}+\cdots+a_{s+n} x^{s+n}, a_{i} \in k, s>0$.

Therefore $v(y)=\inf \left\{v\left(a_{s+i}\right)+(s+i) \pi\right\}, i=0, \cdots, n$, so $v(y)>0$. Thus, $y \in M$ and $P^{*} \subset M$. q.e.d. 
Thus, we have shown that $D$ has the following properties:

(i) $D$ is an intersection of rank one valuation rings.

(ii) Not every nonunit of $D$ is in a minimal prime ideal.

(5.9) Ribenboim, in [14], calls a domain $D$ a "real normal ring with finite character" provided $D$ is an intersection of rank one valuation rings such that every element of $D$ is a nonunit in at most a finite number of these valuation rings. This is a generalization of the notion of "Krull ring', where the valuations involved are also assumed to be discrete. A rank one valuation of the quotient field $K$ of $D$ is called essential by Ribenboim if its valuation ring is a quotient ring of $D$ with respect to a prime ideal of $D$. He then asks whether every real normal ring with finite character is the intersection of the valuation rings of its essential valuations, [14, p. 218]. This is true for Krull rings and is fundamental in the study of such rings.

We have already observed that the $D$ constructed above is a real normal ring with finite character. From property (ii) above, it follows that $D$ is not the intersection of the valuation rings of its essential valuations; for if it were, then every nonunit of $D$ would be in one of the centers, which are necessarily minimal prime ideals of $D\left({ }^{12}\right)$.

\section{REFERENCES}

1. N. Bourbaki, Algèbre commutative, (a) Chapters 5 and 6, 1964, (b) Chapter 7, 1965, Hermann, Paris. 97-103.

2. R. Gilmer and J. Ohm, Integral domains with quotient overrings, Math. Ann. 153 (1964),

3. - Primary ideals and valuation ideals, Trans. Amer. Math. Soc. 117 (1965), 237--250.

4. M. Henriksen, On the ideal structure of the ring of entire functions, Pacific J. Math. 2 (1952), 179-184.

5. - On the prime ideals of the ring of entire functions, Pacific J. Math. 3(1953), 711-720.

6. P. Jaffard, Les systemes d'idéaux, Dunod, Paris, 1960.

7. W. Krull, Uber die Zerlegung der Hauptideale in allgemeinen Ringen, Math. Ann. 105 (1931), 1-14.

8. ——, Allgemeine Bewertungstheorie, J. Reine Angew. Math. 167 (1931), 160-196.

9. —_ Beitrage zur Arithmetik kommutativer Integritatsbereiche. I, Math. Z. 41 (1936), 544-577.

10. - Beitrage zur Arithmetik kommutativer Integritatsbereiche. II, Math. Z. 41 (1936), 665-679.

11. M. Nagata, Local rings, Interscience, New York, 1962.

12. T. Nakayama, On Krull's conjecture concerning completely integrally closed integrity domains. I, Proc. Imp. Acad. Tokyo 18 (1942), 185-187; II, Proc. Imp. Acad. Tokyo 18 (1942), 233-236; III, Proc. Japan Acad.22 (1946), 249-250.

(12) Ribenboim has informed us (August, 1965) that his student M. Griffin has made an exhaustive study of this problem in his May, 1965, doctoral dissertation at Queen's University and that Griffin has obtained a counterexample similar to the one we give here. The question apparently originated with Krull [7, p. 6]. We are also indebted to Ribenboim for making known to us the paper [15] of Samuel. The Bourbaki exercise which we refer to for Theorem 0.1 first appeared there. 
13. P. Ribenboim, Sur une conjecture de Krull en théorie des valuations, Nagoya Math. J. 9 (1955), 87-97.

14. - - Anneaux normaux réels à caractère fini, Summa Brasil. Math. 3 (1956), 213-253.

15. P. Samuel, Méchants anneaux de séries formelles, J. d'Algèbre, Univ. de Clermont, Polycopied notes, 1963, 1-5.

16. G. Schiffels, Graduierte Ringe und Moduln, Bonn. Math. Schr. 11 (1960).

17. A. Seidenberg, Derivations and integral closure, Pacific J. Math. (to appear).

18. O. Zariski and P. Samuel, Commutative algebra, Vol. I, Van Nostrand, New York, 1958.

19. —_ Commutative algebra, Vol. II, Van Nostrand, New York, 1961.

UNIVERSITY OF WISCONSIN,

MADISON, WISCONSIN

UNIVERSITY OF CALIFORNIA,

BERKELEy, CALIFORNIA

Louisiana State University,

Baton Rouge, Louisiana 